\title{
At the tipping point for epigenetic therapies in cancer
}

\author{
Peter A. Jones \\ Departments of Urology and Biochemistry and Molecular Biology, USC Norris Comprehensive Cancer Center, Keck School of Medicine, \\ University of Southern California, Los Angeles, California, USA.
}

\begin{abstract}
The field of epigenetics has exploded in the last two decades, with incredible advances in recent years driven by high-throughput sequencing studies. Cancer cells frequently exhibit marked changes in DNA methylation and histone modification during tumorigenesis and tumor progression. These changes in the cancer epigenome are thought to be important in initiating and maintaining malignancy, and pharmaceutical approaches targeting epigenome-modifying enzymes are an attractive therapeutic strategy. Early successes have been made with DNA-demethylating drugs in hematologic malignancies, and efforts are underway to target additional epigenetic regulators and a broader array of tumor types. The Reviews in this issue of the JCI highlight ongoing efforts in this burgeoning field to translate our understanding of the cancer epigenome into successful interventional strategies in the clinic.
\end{abstract}

The contribution of epigenetic processes, which regulate the packaging of the genome into heritable somatic cell states, to disease pathogenesis has become readily apparent over the last two decades. Indeed, there has been extraordinary growth in the field, with the number of publications discussing epigenetics growing from approximately 100 in 1992 to well over 18,000 in the last year (1). To a large extent, the revolution in epigenetics has been driven by high-throughput sequencing and enhanced computational approaches that allow researchers to examine global changes in gene expression in relation to the status of epigenetic modification. The application of this knowledge to medicine has the potential to transform the treatment and prevention of cancer because pathologic epigenetic gene silencing is an almost universal feature of human malignancies (2). Drugs that target the epigenome are beginning to show traction as viable treatment options for patients with hematologic and solid tumors, and hundreds of clinical trials are underway to explore this new frontier.

Epigenomics, which is a branch of genomics, links the genome to function and can be thought of as software that mediates appropriate gene expression at the right place and time. The stability and heritability of the epigenome is achieved to a large extent by interactions between covalent marks on both the DNA and histone components of chromatin. These marks are applied by enzymes known as "writers," interpreted by proteins called "readers," and removed by other enzymes called "erasers." These enzymes function to communicate to chromatin remodelers, which use ATP or other energy sources to either open or close the DNA so that genes are accessible to the transcriptional machinery.

A completely unexpected outcome of the mutational and epigenetic maps of about 10,000 human tumors produced by The Cancer Genome Atlas (TCGA) Project discussed by Daniel Weisenberger (3) is that many have mutations in the proteins that constitute and modify the epigenome. While the frequencies vary, every tumor without exception has modifications in DNA methylation patterns indicative of an altered epigenome, and the vast majority of tumors harbor both genetic and epigenetic abnormalities.

Conflict of interest: Peter A. Jones is a paid consultant for Astex Pharmaceuticals Inc., Eli Lilly and Company, and Zymo Research Corporation.

Citation for this article: J Clin Invest. 2014;124(1):14-16. doi:10.1172/JCI74145.
There are increasing links to functional interactions between these alterations. This knowledge base has been complemented by the NIH-sponsored ENCODE and Epigenomics Roadmap Projects, which provide us with invaluable maps of how genes are organized and controlled in developing and adult human tissues (4). These next-generation sequencing and bioinformatic approaches have radically transformed our understanding of tumor biology. We now know that changes in gene packaging and thus gene control contribute to cancer and quite possibly to other diseases as well.

Just as genetics is central to the evolution of organisms through the germ line, epigenetics is essential for the inheritance of traits in somatic cells. However unlike gene mutations, which are transmitted through the germ line and therefore require reproductive cycles of many years for mutational consequences to manifest, epigenetic processes including DNA methylation are inherently more plastic, even though these modifications are heritable in somatic cells. This plasticity is readily apparent in the aging epigenome, as discussed by Jean-Pierre Issa (5). These gradual changes in the epigenome, as seen in DNA methylation patterns, could potentially result in the eventual loss and gain of function of key genes essential for the life and death of a cell. This epigenetic drift may well contribute also to the inactivation of key tumor suppressor and other cancer-related genes, resulting ultimately in the formation of cancer as a function of age.

Many epigenetic processes are orchestrated by enzymes and therefore are fundamentally druggable, which provides emerging targets for a whole new range of pharmaceuticals (see Targets of epigenetic therapies in cancer). The first drugs to be approved by the FDA for this purpose were the histone deacetylase inhibitors (HDACis), which by inhibiting the erasers of an active acetyl histone mark can potentially increase the expression of genes silenced by oncogenic epigenetic processes. Allison West and Ricky Johnstone discuss the development of new HDACis, which are beginning to show success in the clinic (6).

The field of epigenetic therapy has been driven to a large extent by the use of the inhibitors of DNA methylation 5-azacytidine (Vidaza) and 5-aza-2'-deoxycytidine (Dacogen), which were synthesized in Prague in the 1960s (7). While we have known for many years that these compounds are powerful inhibitors of DNA methylation and inducers of gene expression and differentiation (8), it has taken 


\section{Targets of epigenetic therapies in cancer}

\section{DNA methyltransferases}

DNA methylation in CPG island promoters is associated with gene repression, and cancer cells frequently exhibit hypermethylation of genes involved in tumor suppression, cell cycle control, and DNA repair. Low-dose, sustained use of DNA methyltransferase (DNMT) inhibitors has shown efficacy, and DNMT inhibitors are approved for use in myelodysplastic syndrome. Next-generation DNMT inhibitors and oral formulations are under development.

\section{Histone deacetylases}

Histone deacetylases (HDACs) are a family of enzymes that remove acetyl groups from histones. HDACs are frequently overexpressed in cancer and are associated with aberrant gene expression. Two HDACis have received FDA approval for refractory cutaneous $\mathrm{T}$ cell lymphoma, and others are in development.

\section{BET proteins}

The BET proteins, including bromodomain-containing 3 (BRD3) and BRD4, bind to acetylated histones to promote transcription. A fusion protein of BRD4 and nuclear protein in testis (NUT) is thought to promote oncogenic gene expression in NUT midline carcinoma (NMC). A BET inhibitor is currently in clinical trials for NMC, and other selective inhibitors have shown activity in preclinical studies.

\section{DOT1L}

DOT1L is a histone methyltransferase that targets histone $\mathrm{H} 3$ at lysine 79 . A fusion protein of mixed-lineage leukemia (MLL) and DOT1L drives altered gene expression in several different leukemias, including MLL and infant leukemia. DOT1L inhibitors are currently in clinical trial in patients with rearrangement of the $M L L$ gene.

\section{EZH2}

EZH2 is the catalytic component of the polycomb repressive complex 2, which mediates gene repression through H3K27 methylation. Oncogenic EZH2 mutations that promote enzymatic activity have been found in diffuse large B cell lymphomas and follicular lymphomas. EZH2 inhibitors have shown preclinical efficacy for lymphomas.

almost 40 years to obtain approval for their use in the clinic, where they are now the standard of care for the treatment of myelodysplastic syndrome (9). The unsung hero in the clinical development of these agents is Lewis Silverman who, along with his colleagues, discusses this arduous process in their Review (10). A key impediment to their success in hematological malignancies was the necessity to use these compounds at low doses to avoid off-target effects. It was largely due to the persistence of Silverman as well as the efforts of other leaders in the field, such as Hagop Kantarjian, Pierre Fenaux, and Michael Lübbert, that clinical success was finally achieved in the face of much skepticism $(9,11,12)$. There are also encouraging developments in the clinical deployment of next-generation DNA methylation inhibitors such as SGI-110 (13).

Another factor delaying the use of these compounds has been the lack of molecular markers that predict the response of patients to DNA demethylation therapy. The current state of the field is discussed by Kirsten Grønbæk and colleagues (14); while there are some promising leads, it is quite clear that we have a long way to go before we can predict which patients are likely to respond to this exciting new therapeutic approach. Much more work is needed to identify and validate appropriate biomarkers, and it is somewhat disappointing that such markers are not readily available even though altered DNA methylation is now used clinically to detect, diagnose, and prognosticate cancer.

The success of epigenetic therapies in hematologic malignancies has rekindled the desire to deploy these compounds in the treatment of solid tumors. Historically these drugs have shown limited efficacy in the treatment of solid tumors, but Stephen Baylin and colleagues review exciting new developments in treating common solid tumors with combinations of DNA methylation and HDACis (15). At present, it appears that epigenetic therapies are unlikely to succeed by themselves and are probably best combined with other modalities, such as standard chemotherapy or immunotherapy. A key factor that makes epigenetic therapies so attractive in these tumors is the realization that cancer is a genomic disease, meaning that these drugs are able to target the cancer genome in its entirety. Thus, the abilities of HDACis and DNA methylation inhibitors to cause global alterations in the epigenome may be a significant advantage. This notion runs counter to approaches using targeted therapies with inhibitors of narrow specificities to acquired mutations, which in principle may be more subject to acquired resistance. Because epigenetic processes can be altered at frequencies several orders of magnitude higher than those associated with genetic mutations, it is very likely that cancer cells exploit epigenetic regulation to activate key cellular pathways that are responsible for cancer cell survival. These pathways may well include drug resistance, inhibition of stem cell differentiation, immune surveillance, and many others. Thus, drugs that target epigenomic processes may have great therapeutic potential in the future.

Robert Campbell and Peter Tummino explore the development of exciting new drugs that target other readers, writers, and erasers, such as enhancer of zeste homolog 2 (EZH2) inhibitors, bromodomain and extra-terminal (BET) inhibitors, and DOT1-like histone H3K79 methyltransferase (DOT1L) inhibitors, and present a discussion of developing new therapies from the perspective of the pharmaceutical industry (16). The plethora of mutations that 
have been observed in genes modifying the epigenome (as discussed by Weisenberger; ref. 3) validate the approach of using inhibitors of epigenetic processes for drug development. Importantly many of the observed mutations occur in enzymes, which are far easier to target than other proteins such as transcription factors. Thus, epigenetic drugs can be utilized to change the transcriptional output of a cancer cell. Almost all of the major pharmaceutical companies and many start-ups have therefore embarked on ambitious and aggressive programs to develop a new series of drugs that should offer a great potential for the next generation of cancer therapeutics.

We are therefore at a tipping point in the area of clinical epigenetics. We have the maps, the assays, the drugs, and some very robust patient responses to encourage us to move aggressively forward in taking advantage of the rampant epigenetic lesions discussed in this series, in order to improve survival of our patients.

\section{Acknowledgments}

This work was supported by grants RO1 CA 083867 and R37 CA 082422 from the NCI and a generous grant from Stand Up to Cancer.

Address correspondence to: Peter A. Jones, 1441 Eastlake Ave., Room 7341, Los Angeles, California 90089-9176, USA. Phone: 323.442.8065; Fax: 323.442.8062; E-mail: pjones@med.usc.edu.
1. Google Scholar. http://scholar.google.com. Accessed October 28, 2013.

2. Shen H, Laird PW. Interplay between the cancer genome and epigenome. Cell. 2013;153(1):38-55.

3. Weisenberger DJ. Characterizing DNA methylation alterations from The Cancer Genome Atlas. J Clin Invest. 2014;124(1):17-23.

4. ENCODE Project Consortium, et al. An integrated encyclopedia of DNA elements in the human genome. Nature. 2012;489(7414):57-74.

5. Issa J-P. Aging and epigenetic drift: a vicious circle. J Clin Invest. 2014;124(1):24-29.

6. West AC, Johnstone RW. New and emerging HDAC inhibitors for cancer treatment. J Clin Invest. 2014; 124(1):30-39.

7. Cihak A, Sorm F. Biochemical effects and metabolic transformation of 5-azacytidine in Escherichia coli. Collect Czech Chem Commun. 1965;30(6):2091-2102.

8. Jones PA, Taylor SM. Cellular differentiation, cytidine analogs and DNA methylation. Cell. 1980; 20(1):85-93.
9. Fenaux P, et al. Efficacy of azacitidine compared with that of conventional care regimens in the treatment of higher-risk myelodysplastic syndromes: a randomised, open-label, phase III study. Lancet Oncol. 2009;10(3):223-232.

10. Navada SC, Steinmann J, Lübbert M, Silverman LR. Clinical development of demethylating agents in hematology. J Clin Invest. 2014; 124(1):40-46.

11. Garcia-Manero G, et al. Randomized open-label phase II study of decitabine in patients with lowor intermediate-risk myelodysplastic syndromes. J Clin Oncol. 2013;31(20):2548-2553.

12. Lübbert $M$, et al. Low-dose decitabine versus best supportive care in elderly patients with intermediate- or high-risk myelodysplastic syndrome (MDS) ineligible for intensive chemotherapy: final results of the randomized phase III study of the European Organisation for Research and Treatment of Cancer Leukemia Group and the German MDS Study Group. J Clin Oncol. 2011;29(15):1987-1996.
13. Kantarjian HM, et al. Results From the Dose Escalation Phase of a Randomized Phase 1-2 First-inHuman (FIH) Study of SGI-110, a Novel Low Volume Stable Subcutaneous (SQ) Second Generation Hypomethylating Agent (HMA) in Patients with Relapsed/Refractory MDS and AML. Presented at: The 54th American Society of Hematology Annual Meeting and Exposition. December 8-11, 2012. Atlanta, Georgia, USA. https://ash.confex. com/ash/2012/webprogram/Paper49626.html. Accessed November 5, 2013

14. Treppendahl MB, Kristensen LS, Grønbæk K. Predicting response to epigenetic therapy. J Clin Invest. 2014;124(1):47-55.

15. Ahuja N, Easwaran H, Baylin SB. Harnessing the potential of epigenetic therapy to target solid tumors. J Clin Invest. 2014;124(1):56-63.

16. Campbell RM, Tummino PJ. Cancer epigenetics drug discovery and development: the challenge of hitting the mark. J Clin Invest. 2014; 124(1):64-69. 